\title{
Clinico-
pathological
conference
}

\section{Pyrexia of undetermined origin in advanced HIV disease}

\author{
C M Tang, C P Conlon, R F Miller
}

\section{Case report (Dr C Tang)}

A 60 year old HIV-1 antibody positive homosexual white man was admitted to hospital in June 1995 for investigation of fever, weight loss and neutropenia. He had had syphilis in 1971 and gonorrhoea in 1975. In 1985 he was found to be hepatitis $B$ immune and in February 1987 was found to be HIV-1 antibody positive having probably been infected with HIV some time in 1985 or 1986. In April 1992 investigations revealed that he had IgG antibodies to toxoplasma and CMV.

The patient lived alone, was a non-smoker and drank occasional alcohol. He denied intravenous drug use and kept no pets. He frequently sailed around the coast of Greece and Turkey. He had travelled widely, in 1986 to Kenya, in 1987 to Tahiti, and throughout the late 1980s until 1994 he had been in Europe mainly in Greece and Turkey. $\mathrm{He}$ was last abroad 10 months before admission. Review of his HIV history showed that he had been well until March 1994 when, because of a falling CD4 lymphocyte count (to $0.12 \times$ $10^{9} / 1$, having been $0.45 \times 10^{9} / 1$ in November 1993), he began primary prophylaxis against Pneumocystis carinii with daily co-trimoxazole. By March 1995 his CD4 count had fallen to $0.02 \times 10^{9} / 1$ so he began zidovudine, $250 \mathrm{mg}$ twice daily. Within 2 weeks he had developed marked neutropenia and the zidovudine was stopped. Despite this he remained variably neutropenic so in May 1995 the co-trimoxazole was stopped and dapsone $100 \mathrm{mg}$ once daily was substituted. In addition he developed a sore mouth with gingivitis. This was treated with metronidazole, co-amoxiclav and fluconazole $200 \mathrm{mg}$ once daily. He developed seborrhoeic dermatitis which was treated with hydrocortisone cream and Balneum bath oil.

On admission to hospital in June 1995 he gave a four week history of fever and night sweats, anorexia and weight loss of approximately $1 \mathrm{~kg}$. On direct inquiry he reported a sensation of epigastric fullness after eating, abdominal bloating and constipation. His gingivitis had persisted despite treatment. On examination he looked well weighing $80 \mathrm{~kg}$. $\mathrm{He}$ was pyrexial with a temperature of $38.8^{\circ} \mathrm{C}$; there was no lymphadenopathy. On the skin a lesion of Kaposi's sarcoma was noted on the left second toe; there were no stigmata of vasculitis. The chest was clear, cardiovascular examination showed that the pulse was 120 /minute and regular and no cardiac murmurs were audible. He had gingivitis and a carious left upper first molar. No Kaposi's sarcoma was seen on the plate. Abdominal examination was normal, with no hepato- splenomegaly nor palpable masses. Neurological examination was unremarkable. Funduscopy with the pupils dilated with tropicamide, showed no retinal abnormalities.

Investigations included urinanalysis, which was normal, a full blood count showing $\mathrm{Hb} 9.2 \mathrm{~g} / \mathrm{dl}$ and WBC $1.29 \times 10^{9} / 1$; the differential count was neutrophils $40 \%$, lymphocytes $37 \%$, monocytes $19 \%$ and eosinophils $1 \%$. The platelet count was $159 \times 10^{\%} / 1$. A CD4 lymphocyte count was $0.02 \times 10^{9} / 1$ (normal 0.35 to $\left.2.2 \times 10^{\%} / 1\right)$. A peripheral blood film showed a normochromic normocytic anaemia and examination of thick blood films revealed no malaria parasites. The ESR was $56 \mathrm{~mm}$ in the first hour and CRP $25 \mathrm{mg} / \mathrm{l}$. Urea and electrolytes and liver function tests were normal except for the GT which was 53 (normal range < 50) IU/1. A cryptococcal latex agglutination (CRAG) test was negative. Other investigations included CMV serology which showed that the patient was latex positive; IgM antibodies were not detected. Toxoplasma serology also showed evidence of IgG antibodies. Syphilis serology $=$ TPHA and FTA positive; RPR negative. An abdominal ultrasound scan was normal. Microscopy and staining of a spontaneously expectorated sample of sputum showed no alcohol or acid fast bacilli (AAFB); subsequent culture was negative for mycobacteria. Three blood cultures showed no growth. A chest radiograph (fig 1) showed coarse reticulonodular shadowing without mediastinal/hilar lymphadenopathy or pleural effusions.

In view of the history of possible gastric outflow obstruction the patient underwent upper gastrointestinal endoscopy. This showed there was spasm of the pylorus with gastric polyps. These were biopsied and histology revealed changes of hyperplasia only. A barium meal examination showed no evidence of obstruction. A course of metronidazole, given for dental sepsis, had no impact on the fever. As the chest radiograph showed a diffuse abnormality the differential diagnosis included infection and tumour. Resting and exercise $\mathrm{SaO}_{2}$ (on air) were normal. A fibreoptic bronchoscopy showed no endobronchial abnormalities. Bronchoalveolar lavage (BAL) was performed from the right middle lobe. Staining of BAL fluid with Papanicolaou showed alveolar macrophages. Methenamine silver and Ziehl Neelsen stains were both negative. The Gram stain showed both Gram positive and Gram negative cocci and rods. Culture of BAL fluid grew alpha haemolytic streptococci, Neisseria spp, Staphylococcus aureus and cytomegalovirus (CMV). \\ Unit, Nuffield
Department of Clinical
Medicine, University \\ John Radcliffe \\ OX3 9DU \\ November 1996
}

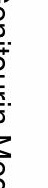
章 


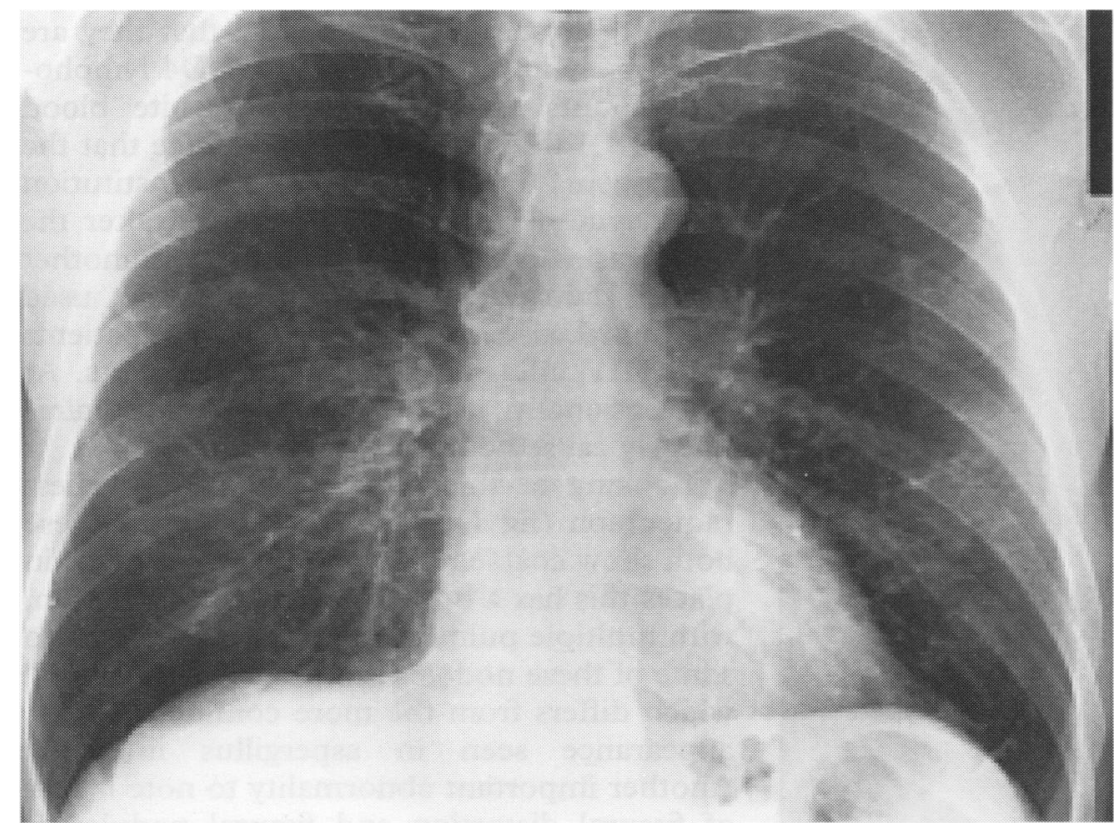

Figure 1 Chest radiograph, taken on admission, showing coarse reticulonodular shadowing. There is no mediastinal/hilar lymphadenopathy and no pleural effusion.

He remained neutropenic and because his fever had not settled, empiric therapy with imipenem and netilmicin was commenced. The next day his left upper first molar was extracted. Following this there was some reduction in the fever (fig 2 ) but it was unclear whether this was due to the extraction or the imipenem and netilmicin. Computed tomography (CT) of the thorax was performed (fig 3). This showed multiple pulmonary nodules some of which had shaggy incomplete haloes adjacent to them. Fissural nodularity and distortion was noted. Within the right middle lobe and lingula the abnormalities had a bronchovascular distribution. Of note there was no hilar or mediastinal lymphadenopathy and no pleural effusions. Within the parenchyma there was no evidence of ground glass shadowing. The CT abnormalities were felt unlikely to be due to $P$ carinii pneumonia or to $\mathrm{CMV}$ pneumonitis. He remained neutropenic so on day 12 a bone marrow aspirate and trephine were performed. This showed reduced erythroid activity and some dysplastic cells were seen. These changes were ascribed to HIV infection. On day 14 of admission a diagnosis was made.

\section{Discussion (Dr R F Miller)}

In summary this is a 60 year old white homosexual male who was infected with HIV approximately 10 years ago; recently his CD4 lymphocyte count has been falling rapidly and it is now only $0.02 \times 10^{\%} / 1$. He is therefore profoundly immunosuppressed. $\mathrm{He}$ was admitted for investigation of fever, anorexia, abdominal bloating and constipation. Initial examination shows gingivitis and a Kaposi's sarcoma lesion on the skin. There is a striking absence of lymphadenopathy and organomegaly. Investigations show anaemia, neutropenia and borderline thrombocytopenia. The chest radiograph and CT show abnormalities but no diagnosis has been reached by day 13. A trial of empiric antibiotics has little impact on his symptoms. This patient has a pyrexia of undetermined origin (PUO). PUO is the term used to describe patients with a prolonged febrile illness in whom no cause is found after a period of inpatient investigation. The diagnostic criteria are an illness of $>3$ weeks' duration, documented fever $>38.3^{\circ} \mathrm{C}$, recorded on several occasions, and no cause identified after one week of investigation in hospital. ${ }^{12}$ Studies of PUO in the general population show that the majority of cases are due to infection, neoplasia or connective tissue disorders. ${ }^{1-6}$ Studies of PUO in HIV infected patients show that the majority of episodes are due to infection or tumour, and only rarely is PUO caused by HIV itself. ${ }^{7}{ }^{9}$

Moving now to this case there is a wide dif-
Figure 2 Patient' temperature chart. Despite empirical antibiotics and dental extraction the fever persists.

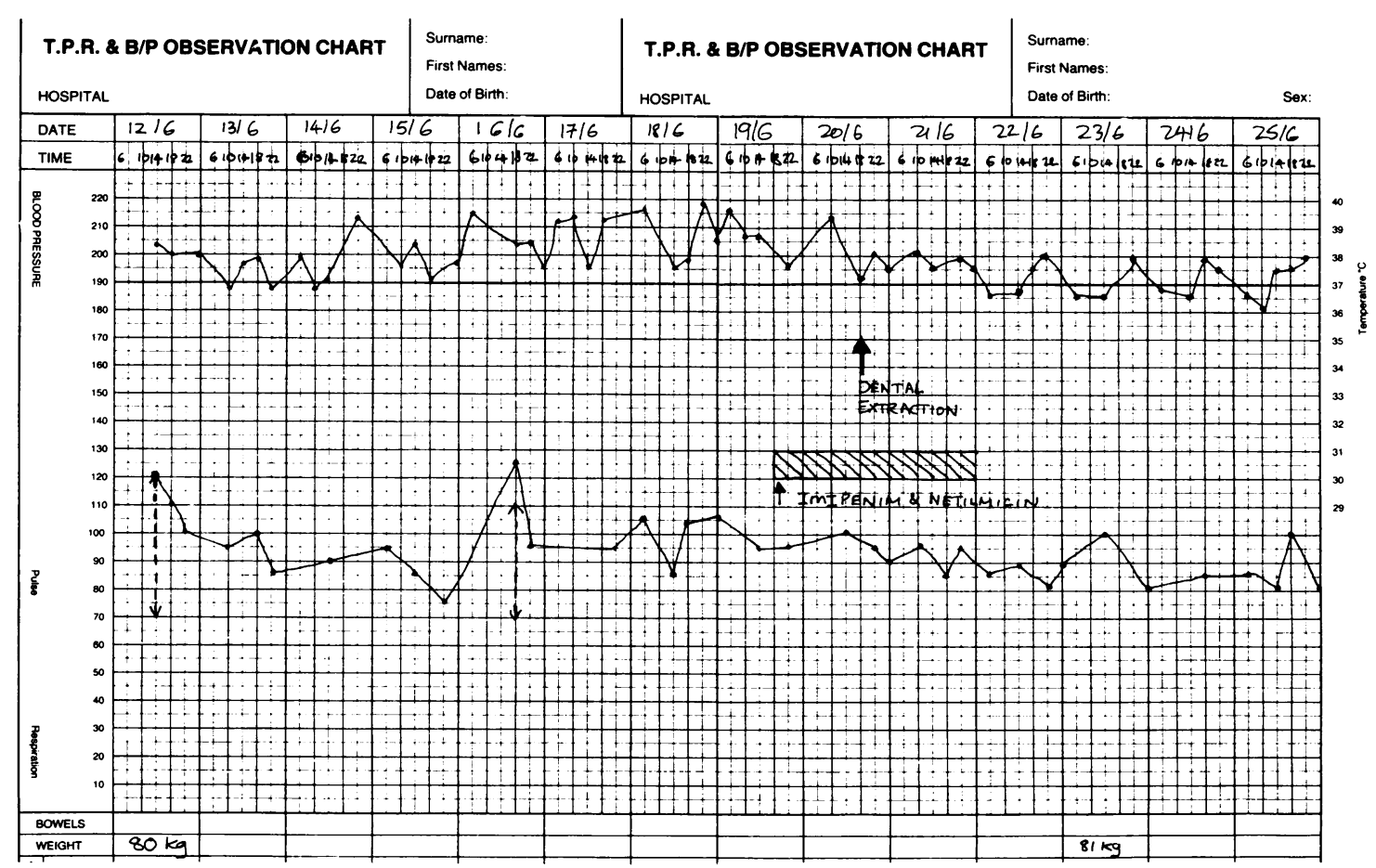




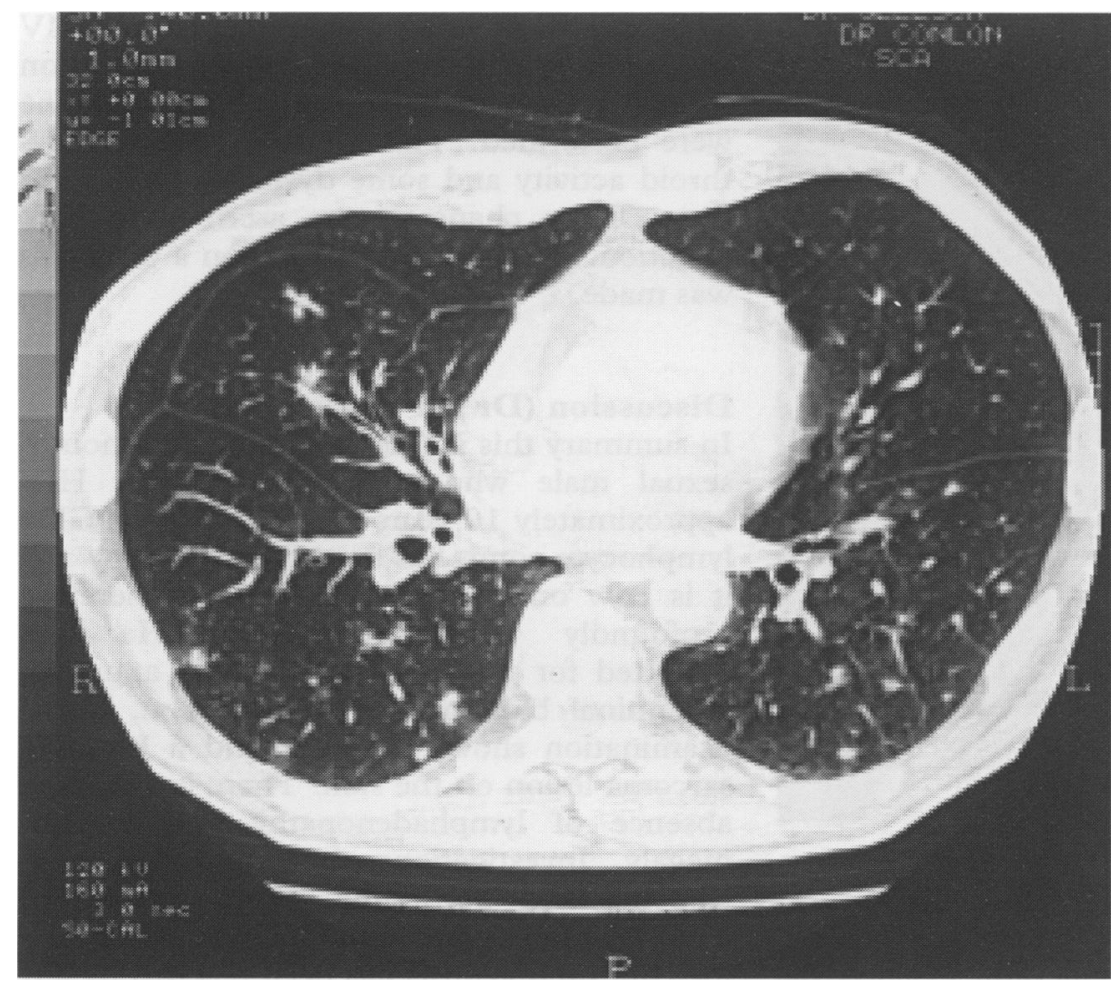

Figure 3 CT scan of the thorax showing multiple intrapulmonary nodules.

ferential diagnosis to consider (table 1). I think that it is unlikely that this patient's presentation is due to HIV "constitutional disease". Although fever, weight loss and malaise are commonly caused by HIV infection they are very non-specific symptoms. Constitutional disease also occurs more commonly early in the HIV disease course, typically when the CD4 lymphocyte count falls below $0.30 \times$ $10 \%$. Could an adverse drug reaction account for this patient's presentation? Adverse drug reactions are much more common in HIV infected patients than in the general population. ${ }^{10}$ Co-trimoxazole even at doses used for prophylaxis is particularly associated with adverse reactions including fever, rash, and leucopenia/anaemia. ${ }^{11}$ In this case the cotrimoxazole had been stopped because of neutropenia and dapsone substituted. Despite this neutropenia persisted and on this background fever developed. Zidovudine, particularly when used at dose of 1.0 to $1.2 \mathrm{~g}$ per day not infrequently causes anaemia/neutropenia. From the Concorde study of immediate versus deferred zidovudine in the treatment of early HIV infection we know the onset of these adverse reactions occurs most often 8 to 12

Table 1 Differential diagnosis of PUO in AIDS

$\begin{array}{ll}\begin{array}{r}\text { HIV related } \\ \text { Infection }\end{array} & \begin{array}{l}\text { bacterial } \\ \text { mycobacterial } \\ \text { viral } \\ \text { fungal } \\ \text { protozoal } \\ \text { lymphoma }\end{array} \\ \text { Kamosi's sarcoma } \\ \text { Tumour } \\ \text { Adverse drug reaction } \\ \text { Due to HIV itself-HIV “constitutional disease" } \\ \text { Not related to HIV } \\ \text { Infection } \\ \text { Tumour } \\ \text { Connective tissue disorder } \\ \text { Factitious fever }\end{array}$

weeks after starting therapy and that they are more likely in patients with low CD4 lymphocyte counts and low baseline white blood counts. ${ }^{12} I$ think that it is very strange that the neutropenia occurred so soon after institution of zidovudine therapy and persisted after the drug was stopped. I wonder if there is another $\Phi$ cause? Zidovudine and co-trimoxazole, used $\stackrel{\mathbb{D}}{=}$ as prophylaxis, are commonly given to patients with HIV infection and low CD4 counts. At conventionally used doses they only infre- $\frac{2}{\infty}$ quently cause neutropenia. ${ }^{13}$

Looking at this patient's admission chest $\overrightarrow{\bar{F}}$ radiograph (fig 1) and CT scan (fig 3) these $\stackrel{0}{?}$ both show coarse reticulonodular infiltrates; in $\frac{\text { ? }}{5}$ places this has a bronchovascular distribution, $\frac{5}{\bar{m}}$ with multiple pulmonary nodules. Adjacent to $\widehat{\nabla}$ some of these nodules there is a shaggy "halo" 을 which differs from the more confluent "halo" क appearance seen in aspergillus infection. $\vec{\circ}$ Another important abnormality to note is that $\overrightarrow{\vec{\omega}}$ of fissural distortion and fissural nodularity, $\frac{\sigma}{\omega}$ this finding is pathognomonic for pulmonary Kaposi's sarcoma. I note that there is no $\omega$ "ground glass" shadowing which makes $P+$ carinii pneumonia and CMV pneumonitis ${ }_{\infty}^{\infty}$

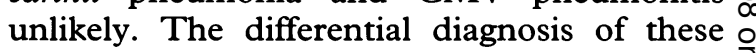
imaging abnormalities includes Kaposi's sarcoma, fungal infection, such as histoplasmosis (although the absence of lymphadenopathy is against this diagnosis) and mycobacterial $\stackrel{\complement}{\hookrightarrow}$ infection. I am not put off a diagnosis of pul- $\vec{\bullet}$ monary Kaposi's even though no palatal or tracheobronchial lesions were seen. Palatal i lesions of Kaposi's sarcoma strongly predict for the presence of visceral disease but pulmonary (and gastrointestinal) Kaposi's may occur without cutaneous and palatal lesions. $\stackrel{\mathbb{\perp}}{\propto}$ Within the lung parenchymal and pleural $\overrightarrow{\overrightarrow{0}}$ Kaposi's may occur without tracheobronchial $\frac{3}{3}$ disease or hilar/mediastinal lymphadenopathy. ${ }^{14}$ There are reports of Kaposi's sarcoma presenting with unexplained fever but I am unaware of any association with neutropenia. ${ }^{15}$ It is important to remember that this patient has been infected with HIV for almost a $\delta$ decade and is profoundly immunosuppressed and pancytopenic thus we must consider lym- 운 phoma as a possible cause. Against this is the absence of superficial and intrabdominal or thoracic lymphadenopathy. The bone marrow examination has not revealed diagnostic infor- $N$ mation but the diagnosis of lymphoma is not $\mathrm{N}$ excluded by a negative bone marrow aspirate. A whole body gallium-67 citrate radionuclide 0 scan would have been a useful test to consider $\stackrel{\oplus}{\Phi}$ in this patient. Gallium-67 accumulates at sites of inflammation caused by infection or $\frac{0}{0}$ lymphoma ${ }^{16}$ and does not accumulate in $\frac{0}{\Phi}$ Kaposi's sarcoma lesions. Hence, the absence $\stackrel{?}{\mathbb{D}}$ of pulmonary accumulation in the presence of an abnormal chest radiograph would favour a diagnosis of Kaposi's sarcoma. The absence of $\frac{0}{0}$ accumulation either within the lung or elsewhere together with the absence of lymphadenopathy or organomegaly would make lymphoma very unlikely. ${ }^{17}$ Lung cancer is no more frequent in HIV infected patients compared with the general population but when it occurs may have a more aggressive course. 
Table 2 Clinical features of disseminated histoplasmosis in AIDS patients

\begin{tabular}{ll}
\hline & Number of patients (\%) \\
\hline Symptoms & \\
fever & 81 \\
weight loss $(>4.5 \mathrm{~kg})$ & 52 \\
cough & 21 \\
night sweats & 19 \\
diarrhoea & 19 \\
dyspnoea & 13 \\
Signs & \\
splenomegaly & 31 \\
hepatomegaly & 19 \\
lymphadenopathy & 19 \\
$\quad$ rash & 10 \\
Investigations & \\
$\quad$ abnormal chest radiograph & 52 \\
(infiltates or lymphadenopathy) & 27 \\
thrombocytopenia & 23 \\
anaemia & 21 \\
\hline leucopenia & \\
\hline Data from Johnson et al. ${ }^{19}$ &
\end{tabular}

Data from Johnson et al. ${ }^{19}$

Against this diagnosis are the negative results from bronchoscopy and lavage and bone marrow sampling and the absence of specific features on CT and abdominal ultrasonography in this patient.

What about bacterial infections as a cause of this man's symptoms? He has several possible sources, including dental sepsis, which is more common in HIV infected patients with low CD4 counts. Dental extraction and antibiotics for gingivitis did not affect the symptoms. An empirical course of antibiotics for "neutropenic fever" similarly had little impact. I think the mixed micro-organisms from the BAL fluid represent only naso/oropharyngeal flora.

Staining of sputum, BAL fluid and blood was negative for AAFB. At this stage of HIV infection Mycobacterium tuberculosis is frequently a disseminated infection and it is known that up to one third of those with pulmonary tuberculosis have smear negative sputum or BAL fluid yet are subsequently positive by culture. ${ }^{18}$ I would be very keen to know the results of BAL culture for mycobacteria and of special staining of the bone marrow aspirate as this might also show AAFB. Culture of this would identify whether this is Mycobacterium tuberculosis or an atypical mycobacteria such as $M$ avium-intracellulare. In favour of $M$ aviumintracellulare is the abdominal pain but against is the absence of intra-abdominal lymphadenopathy or organomegaly and the fact that abdominal $M$ avium-intracellulare infection is more commonly associated with diarrhoea.

This patient has serological evidence of past CMV infection. Up to $90 \%$ of homosexual HIV infected men are known to intermittently shed CMV in urine, semen, blood and respira-

Table 3 Clinical features of visceral leishmaniasis in AIDS patients

\begin{tabular}{llll}
\hline & \multicolumn{2}{l}{ Number of patients (\%) } \\
\cline { 2 - 4 } & Altes et al $^{21}$ & Montalban et al ${ }^{22}$ & Berenguer et al $^{23}$ \\
\hline $\begin{array}{l}\text { Symptoms } \\
\text { fever }\end{array}$ weight loss & 91 & 91 & 66 \\
$\begin{array}{l}\text { Signs } \\
\text { splenomegaly }\end{array}$ & 95 & - & 55 \\
$\begin{array}{l}\text { Investigations } \\
\quad \text { neutropenia }\end{array}$ & 91 & 87 & 66 \\
$\begin{array}{l}\text { thrombocytopenia } \\
\text { pancytopenia }\end{array}$ & 74 & - & 44 \\
\hline
\end{tabular}

tory secretions. CMV inclusions were identified in the BAL fluid. I am not convinced that this is of clinical significance as this is a not uncommon finding even in the absence of CMV associated pneumonitis.

This man has a long travel history. Of note he has travelled widely and repeatedly around the eastern Mediterranean in recent years and I just wonder about a fungal infection such as histoplasmosis. Many features of this patient's presentation are in keeping with disseminated histoplasmosis (table 2) ${ }^{19}$; however, the absence of hepatosplenomegaly or lymphadenopathy are atypical. ${ }^{19}$ Returning to the bone marrow investigation once more I would be interested to see the results of special staining for fungi. I think cryptococcal infection is unlikely, the organism was not grown from BAL fluid or blood, and the CRAG test is negative in blood. Aspergillus should be considered in view of the neutropenia, but this organism was not identified in BAL fluid and the CT appearances are atypical.

Could this man have malaria? He travelled to Kenya 10 years ago but gives no clinical history of malaria at the time or subsequently. Thick films of peripheral blood are negative for malaria parasites. What about other protozoa such as leishmaniasis? The patient has prolonged and recurrent travel through an endemic area. In this geographical region Leishmania infantum is the causative organism and the reservoir is in dogs and foxes and the vector is the sand fly. It is estimated that in Spain $50 \%$ of adults with leishmaniasis are HIV infected and that $3 \%$ of all HIV infected adults will develop leishmaniasis..$^{20}$ In addition to the travel history several other features are suggestive of leishmaniasis (table 3).$^{920-23} \mathrm{I}$ am not put off this diagnosis by the absence of splenomegaly. Although two studies of leishmaniasis in HIV infected patients have shown splenomegaly to be an almost universal finding, ${ }^{2122}$ a study by Berenguer et $a l^{23}$ found splenomegaly in only two thirds of cases. The diagnosis of leishmaniasis is most often made by staining of a splenic or bone marrow aspirate and subsequent culture. The study by Berenguer et $a l^{23}$ was noteworthy as two thirds of the patients in this series had negative results from staining of bone marrow aspirate yet were subsequently culture positive for leishmania and half of those without a positive bone marrow stain for leishmania also had no splenomegaly. So I think that a diagnosis of leishmaniasis remains a distinct possibility in this man. Yet again I found myself coming back to the bone marrow and I wonder what special stains and culture revealed.

In conclusion I think that this profoundly immunosuppressed HIV infected white male's history has several important features. The travel history, the cutaneous Kaposi's sarcoma, the absence of lymphadenopathy and organomegaly, the pancytopenia and the chest radiographic abnormalities. I think the diagnosis made on day 14 was probably from the bone marrow aspirate and I just wonder what the special bone marrow stains revealed. My differential diagnosis is: 
1 AIDS

2 Kaposi's sarcoma, of skin (and probably the lung also)

3 Visceral leishmaniasis or

4 Disseminated histoplasmosis.

\section{Pathology and discussion (Dr C P Conlon)}

The bone marrow aspirate and trephine showed many monocytes and macrophages packed with numerous leishmania promastigotes (fig 4) confirming a diagnosis of visceral leishmaniasis. He was started on sodium stibogluconate, $850 \mathrm{mg}$ daily intravenously, which he received for 28 days. His constitutional symptoms gradually improved, his fever settled and his pancytopenia resolved over the period of treatment. A repeat bone marrow examination 2 months after stopping treatment showed dysplastic changes typical of HIV infection but no evidence of residual leishmaniasis.

Visceral leishmaniasis is an increasingly recognised complication of HIV infection in areas where the two infections co-exist but is also seen in HIV positive individuals who visit areas where leishmania is endemic. ${ }^{24}$ This patient regularly visited parts of the Mediterranean coast where Leishmania infantum is known to be endemic. This parasite may cause cutaneous leishmaniasis but sometimes leads to visceral leishmaniasis in immunocompetent people. The parasite is inoculated into the dermis by the bite of the female phlebotomine sand fly and is rapidly taken up by macrophages in the skin. Normally, the parasite is either destroyed by the macrophages or is contained in the skin, leading to the lesions of cutaneous leishmaniasis. In immunosuppressed individuals (and some immunocompetent ones) such local control fails and the parasite spreads throughout the reticuloendothelial system, resulting in visceral leishmaniasis. Typically, patients with visceral leishmaniasis present with fever, some weight loss, lymphadenopathy and hepatosplenomegaly. Most have a moderate pancytopenia, reflecting bone marrow infiltration with parasite laden macrophages, and a marked hypergammaglobulinaemia. Lung infiltrates and nodules have been described. The clinical features may be modified in patients with HIV so that lymphadenopathy is

Figure 4 Bone marrow Giemsa stain, magnification $\times 600$ promastigotes of Leishmania infantum are evident less common and up to $30 \%$ have no evidence of hepatosplenomegaly. ${ }^{23}$ Fever with pancytopenia may be the only features, as they were in this case. The diagnosis is made by microscopy and culture of a bone marrow aspirate or, if there is splenomegaly, by splenic puncture. Serology is unreliable, particularly in relapses, but molecular methods of diagnosis may be helpful. ${ }^{25}$

Visceral leishmaniasis is usually treated with pentavalent antimony compounds, such as sodium stibogluconate or meglumine antimo- $\stackrel{\mathbb{Q}}{\mathrm{Q}}$ niate. Other drugs that have been used successfully include amphotericin B, pentamidine, allopurinol and paromomycin. The optimal treatment for visceral leishmaniasis in the context of HIV infection is not known. Failure of antimony compounds has been described and successes have been reported with amphotericin B, particularly in liposomal formula- $\vec{o}$ tions. ${ }^{21}$ However, no formal randomised trials $\overrightarrow{\vec{H}}$ of these treatment have been done and relapses after apparent successful treatment $\stackrel{\text { on }}{=}$ with both drugs are common in HIV disease..$^{26} \omega$

We elected to treat our patient with sodium stibogluconate on the grounds that it was ${ }_{\infty}^{\omega}$ likely to be effective, he was generally fairly well with normal renal function and there were no good data in favour of the considerably more expensive liposomal amphotericin B cे preparations. After his good response to therapy we considered maintenance treatment $\vec{\bullet}$ with sodium stibogluconate but, because of the risk of cumulative toxicity, we decided to wait until there was a clinical relapse. Six months after being treated for leishmaniasis the patient died of progressive Kaposi's sarcoma and terminal CMV encephalitis. He had no clinical evidence of recurrent leishmaniasis but a postmortem examination was not performed.

\section{Final diagnosis \\ 1 AIDS}

2 Visceral leishmaniasis

3 Kaposi's sarcoma

\section{Key points}

1 Pyrexia of undetermined origin in HIV infected patients is only rarely due to HIV "constitutional" disease.

2 Obtaining a detailed travel history is important in any patient with unexplained fever.

3 Leishmaniasis may occur, without splenomegaly, in HIV infected patients.

This CPC was presented as an "unseen case" at the staff round on 14 March 1996 at the John Radcliffe Hospital, Oxford. We 0 thank Dr A Berendt for allowing us to report this case and Jane Rutherford for typing the manuscript.

1 Petersdorf RG, Beeson PB. Fever of unexplained origin. Medicine 1961;40:1-30.

2 Shoen RP, Van Ommen RA. Fever of obscure origin. $A m \mathcal{F}$ Med 1963;34:486-99.

3 Howard P, Hahn HH, Palmer PL, Hardin WJ. Fever of unknown origin: a prospective study of 105 patients. Tex Med 1977;73:56-9.

4 Larson EB, Featherstone HJ, Petersdorf RG. Fever of undetermined origin: diagnosis and follow up of 100 cases 1970-80. Medicine 1982;61:269-92. 
5 Barbado FJ, Vazques J, Pena JM. Fever of unknown origin: a survey on 133 patients. $\mathcal{F}$ Med $1984 ; 15: 185-92$

6 Knockaert DC, Vanneste LJ, Vanneste SB, Bobbaers HJ. Fever of unknown origin in the 1980s: an update of the diagnostic spectrum. Arch Intern Med 1992;152:51-2.

7 Miller RF, Hingorami AD, Foley NM. Pyrexia of undetermined origin in patients with human immunodeficiency virus and AIDS. Int $\mathcal{F}$ STD AIDS 1996;7:170-5.

8 Bissuel F, Leport C, Peronne C, Longuet P, Vilde JL. Fever of unknown origin in HIV-infected patients: a critical analysis of a retrospective series of 57 cases. $\mathcal{F}$ Intern Med 1994;236:529-35.

9 Miralles P, Morenos S, Perez-Tascon M, Cosin J, Diaz $\mathrm{MD}$, Bouza E. Fever of uncertain origin in patients MD, Bouza E. Fever of uncertain origin in patients infected with the human

10 Harb GE, Alldredge BK, Coleman R, Jacobson MA Pharmacoepidemiology of adverse drug reactions in hospitalised patients with human immunodeficiency virus disease. $\mathcal{F}$ Acq Immun Def Syn 1993;6:919-22.

11 Koopmans PP, van der Ven AJAM, Vree TB, van der Meer JWM. Pathogenesis of hypersensitivity reactions to drugs in patients with HIV infection: allergic or toxic. AIDS 1995;9:217-22.

12 Concorde: MRC/ANRS randomised double-blind controlled trial of immediate and deferred zidovudine in symptom free HIV infection. Concorde Coordinating symptom free HIV infection. Concor

13 Hardy WD, Feinberg J, Finkelstein DM, Power ME, He W, Kaczka C, et al. A controlled trial of trimethoprimsulfamethoxazole or aerosolized pentamidine for secondary prophylaxis of Pneumocystis carinii pneumonia in patients with the acquired immunodeficiency syndrome. N Engl f Med 1992;327:1842-8.

14 Miller RF, Tomlinson MC, Cottrill CP, Donald JJ, Spittle MF, Semple SJG. Bronchopulmonary Kaposi's sarcoma in patients with AIDS. Thorax 1992;47:721-5.

15 Bach MC, Bagwell SP, Fanning JP. Primary pulmonary Kaposi's sarcoma in the acquired immunodeficiency syndrome: a cause of persistent pyrexia. Am 7 Med 1988; 85:274.

16 Hilson AJW, Maisey M. Gallium-67 scanning in pyrexia of unknown origin. $B M 7$ 1979;2:1330-1.

17 Buscombe JR, Oyen WJG, Corstens FHM, Ell PJ, Miller RF. Localisation of infection in HIV antibody positive patients with fever: comparison of the efficacy of gallium 67 citrate and radiolabelled human IgG (HIG). Clin Nucl Med 1995;20:334-9.

18 Smith RL, Yew K, Berkowitz KA, Aranda CP. Factors affecting the yield of acid-fast sputum smears in patients with HIV and tuberculosis. Chest 1994;106:684-6.

19 Johnson PC, Khardouri N, Najiar AF, Butt F, Mansel PWA, Sarosi GA. Progressive disseminated histoplasmosis in patients with acquired immunodeficiency syndrome. in patients with acquired

20 Alvar J, Gutierrez-Solar B, Molina R, Lopez-Valez R, Garcia-Camacho A, Martinez P, et al. Prevalence of Leishmania infection among AIDS patients. Lancet 1992 339:1427.

21 Altes J, Salas A, Riera M, Udino M, Galmés A, Balauzab J, et al. Visceral leishmaniasis: another HIV-associated opportunistic infection? Report of eight cases and review of the literature. AIDS 1991;5:201-7.

22 Montalban V, Calleja JL, Erice A, Laguna F, Clotet B, Podzamczer $\mathrm{D}$, et al. Visceral Leishmaniasis in patients infected with human immunodeficiency virus. 7 Infect 1990;21:261-70.

23 Berenguer J, Moreno S, Cercenado E, Bernaldo de Quiros B, Garcia de la Fuente A, Bouza E. Visceral leishmaniasis B, Garcia de la Fuente A, Bouza E. Visceral leishmaniasis in patients infected with human immuno

24 Peters BS, Fish D, Golden R, Evans DA, Bryceson AD Pinching AJ. Visceral leishmaniasis in HIV infection and AIDS: clinical features and response to therapy. $Q \mathcal{F ~ M e d}$ 1990;77:1101-11

25 Piarroux R, Gambarelli F, Dumon H, Fontes M, Dunan S, Mary C, et al. Comparison of PCR with direct examination of bone marrow aspiration, myeloculture, and serology for diagnosis of visceral leishmaniasis in immunocompromised patients. F Clin Microbiol 1994;32:746-9.

26 Davidson RN, Russo R. Relapse of visceral leishmaniasis in patients who were coinfected with human immunodeficiency virus and who received treatment with liposomal amphotericin B. Clin Inf Dis 1994;19:560. 